\title{
Cáncer testicular: incidencia, epidemiología y etiología. Cinco años de experiencia en el Hospital General de México Dr. Eduardo Liceaga
}

\author{
Testicular cancer: Incidence, epidemiology and etiology. \\ Five years of experience at the Hospital General de México \\ Dr. Eduardo Liceaga
}

Ángel Gurrola-Ortega, Juan Eduardo Sánchez-Núñez, Hugo Rivera-Astorga, Jorge Esteban Magaña-González, Roberto Carlos Sarabia-Estrada, Leopoldo Mateo Garduño-Arteaga, Hugo Arturo Manzanilla-García, Jorge Jaspersen-Gastelum

\begin{abstract}
Resumen
ANTECEDENTES: El cáncer testicular representa 5\% de los tumores que aparecen durante la edad reproductiva del varón, aunque a últimas fechas se está registrando un incremento en su incidencia. El diagnóstico se establece por clínica, marcadores tumorales y ultrasonido testicular. La estirpe histológica es la base para establecer el tratamiento y estimar el pronóstico.

OBJETIVO: Reportar las características epidemiológicas, histopatológicas y etiológicas del cáncer testicular en un hospital de tercer nivel.

MATERIAL Y MÉTODOS: Estudio observacional, descriptivo y retrospectivo efectuado en pacientes con diagnóstico de cáncer testicular de cualquier estirpe histológica atendidos entre 2012 y 2017 en el servicio de Urología del Hospital General de México. La información de las variables cualitativas se resumió como frecuencias simples y relativas en porcentaje. La variabilidad de las frecuencias se obtuvo mediante IC95\%. La edad se resumió en mediana y valores máximos y mínimos. Se utilizó $\chi^{2}$ para contrastar las diferencias entre los tipos de tumores según las variables analizadas y prueba de Kruskal-Wallis.

RESULTADOS: Se estudiaron 142 pacientes; en $44.4 \%$ se encontraron tumores de células germinales mixtas y en $43.7 \%$ seminomas clásicos. En $50 \%$ de los casos los tumores aparecieron entre los 20 y 30 años (media 35.5), con una lateralidad: 56.3\% izquierdo y $43.7 \%$ derecho. En cuanto a la diseminación linfática $(\mathrm{N})$ los tumores seminomatosos tuvieron estadificación N0 en $80.6 \%$ de los casos $(n=50)$ y $95.2 \%$ $(\mathrm{n}=59)$ no tuvieron mestástasis pulmonares $\mathrm{u}$ otras al momento del diagnóstico. La estirpe no seminomatosa (tumores germinales de células mixtas) fue estadio N0, M0 en $57.2 \%(n=36)$ y $87.3 \%(n=55)$ de los casos, respectivamente. Existe un discreto aumento en la incidencia de esta patología en los últimos dos años.

CONCLUSIONES: Pese a los resultados obtenidos, consideramos que se requiere otra metodología de investigación para determinar la causalidad de la presentación del cáncer testicular en nuestra población.

PALABRAS CLAVE: Cáncer testicular; marcadores tumorales; México; tumores de células germinales; seminomas; tumores seminomatosos.
\end{abstract}

Abstract

BACKGROUND: Testicular cancer accounts for $5 \%$ of the tumors in reproductive-age men and there has recently been an increase in its incidence. Diagnosis is made through clinical evaluation, tumor markers, and testicular ultrasound. Histologic strain determines treatment response and outcome.

OBJECTIVE: To report the epidemiologic, histopathologic, and etiologic characteristics of testicular cancer at a tertiary care hospital within the time frame of 2012-2017.
${ }^{1}$ Servicio de Urología, Hospital General de México Dr Eduardo Liceaga, Ciudad de México.

Recibido: julio 2018

Aceptado: agosto 2018

Correspondencia

Ángel Gurrola Ortega

urodgo_gurrola@hotmail.com

Este artículo debe citarse como Gurrola-Ortega A, Sánchez-Núñez JE, Rivera-Astorga H, Magaña-González JE, Sarabia-Estrada RC, Garduño-Arteaga LM, Manzanilla-García HA, JaspersenGastelum J. Cáncer testicular: incidencia, epidemiología y etiología. Cinco años de experiencia en el Hospital General de México Dr. Eduardo Liceaga. Rev Mex Urol. 2018 sept-oct;78(5):347-353. DOI: https://doi.org/10.24245/revmexurol.v78i5.2408 
MATERIALS AND METHODS: A retrospective, observational, and descriptive study was conducted on patients with the diagnosis of testicular cancer of any histologic strain seen at the urology service of the Hospital General de México within the time frame of 2012 to 2017 . The qualitative variables were expressed as simple, relative frequencies in percentages. Frequency variability was obtained through the $95 \% \mathrm{Cl}$. Age were expressed in median and maximum and minimum values. The chi-square test and the Kruskal-Wallis test were employed to contrast the differences between tumor types, according to the variables analyzed.

RESULTS: A total of 142 patients were studied, $44.4 \%$ of whom presented with mixed germ cell tumors and $43.7 \%$ with classic seminomas. Fifty percent of the patients presented with the disease at 20 to 30 years of age (mean 35.5). With respect to laterality, $56.3 \%$ of the tumors were on the left side and $43.7 \%$ on the right. In relation to lymph node dissemination $(N)$ of the seminomatous tumors, $80.6 \%$ of the cases $(n=50)$ were stage N0 and $95.2 \%(n=59)$ did not present with pulmonary metastasis, or any other, at the time of diagnosis. Of the nonseminomatous tumors (mixed germ cell tumors), $57.2 \%(n=36)$ were stage N0 and $87.3 \%(n=55)$ were MO. There was a slight increase in testicular cancer in the last two years.

CONCLUSIONS: Despite our results, we believe a different study methodology is required to determine the cause of testicular cancer in our population.

KEYWORDS: Testicular cancer; Tumor markers; Mexico; Germ cell tumors; Seminomas; Seminomatous tumors

\section{ANTECEDENTES}

El cáncer testicular es la neoplasia más común en el varón durante su edad reproductiva, representa 5\% de las neoplasias sólidas que aparecen entre los 18 y 35 años, ${ }^{1,2}$ con otro pico de incidencia entre los 60 y 70 años. En las últimas cuatro décadas la incidencia se incrementó de 5.7 a 6.8 casos por cada 100,000 habitantes. ${ }^{3-6}$ Desde luego que el incremento puede adjudicarse a dos posibilidades: mayor sensibilidad y especificidad de los métodos diagnósticos o aumento de la exposición a factores de riesgo. ${ }^{2}$

Para 2012, en América Latina, la Agencia Internacional de Investigación del Cáncer de la Organización Mundial de la Salud estimó una incidencia de esta enfermedad de 2.6 por cada 100,000 habitantes..$^{4,7}$ En 2016, Estados Unidos estimó 8,700 nuevos casos de cáncer testicular y reportó 380 defunciones por esta causa. ${ }^{7,8}$

Los factores de riesgo más estrechamente vinculados son: criptorquidia, orquitis viral, exposición gestacional al dietilestilbestrol y tener un familiar, en primer grado, con cáncer testicular. ${ }^{8,9}$ Desde el punto de vista clínico los tumores testiculares son discretamente más frecuentes del lado derecho (57\%) que en el izquierdo $(43 \%)$. 3,10

La presentación habitual es: aumento de tamaño y consistencia del parénquima gonadal, ${ }^{11}$ dolor, pesantez en el hipogastrio, ginecomastia y manifestaciones endocrinológicas (según la estirpe histológica). El diagnóstico se establece de acuerdo con la clínica, exploración, concentraciones de marcadores tumorales (alfa-feto proteína - $\alpha-\mathrm{FP}$, deshidrogenasa láctica - $\mathrm{DHL}$, gonadotropina coriónica humana - $\beta-\mathrm{GCH}$ ) y el ultrasonido testicular. ${ }^{12,13,14}$ Según la National Comprehensive Cancer Network (Cuadro 1) para la estadificación clínica y el seguimiento es indispensable la tomografía abdomino pélvica y la telerradiografía de tórax. ${ }^{13,15,16}$

Desde el punto de vista histológico el cáncer testicular se clasifica en tumores de células ger- 
Cuadro 1. Estadios clínicos del cáncer testicular, NCCN

\begin{tabular}{|c|c|c|c|c|}
\hline Estadio & Tumor & Ganglios & Metástasis & Marcadores \\
\hline Estadio 0 & pTis & No & MO & SO, Sx \\
\hline Estadio I & pT1-T4 & No & MO & Sx \\
\hline $\begin{array}{l}\text { Estadio } \\
\text { IA }\end{array}$ & Pt1 & No & MO & So \\
\hline $\begin{array}{l}\text { Estadio } \\
\text { IB }\end{array}$ & pT2-pT4 & No & MO & So \\
\hline $\begin{array}{l}\text { Estadio } \\
\text { IS }\end{array}$ & $\begin{array}{c}\text { Cualquier } \\
T / T x\end{array}$ & No & Mo & S1-3 \\
\hline Estadio II & $\begin{array}{c}\text { Cualquier } \\
\mathrm{T} / \mathrm{Tx}\end{array}$ & N1-N3 & MO & Sx \\
\hline \multirow[t]{2}{*}{$\begin{array}{l}\text { Estadio } \\
\text { IIA }\end{array}$} & $\begin{array}{c}\text { Cualquier } \\
\text { T/TX }\end{array}$ & N1 & MO & SO \\
\hline & $\begin{array}{c}\text { Cualquier } \\
\mathrm{T} / \mathrm{Tx}\end{array}$ & N1 & Mo & S1 \\
\hline \multirow[t]{2}{*}{$\begin{array}{l}\text { Estadio } \\
\text { IIB }\end{array}$} & $\begin{array}{c}\text { Cualquier } \\
\mathrm{T} / \mathrm{TX}\end{array}$ & N2 & Mo & So \\
\hline & $\begin{array}{c}\text { Cualquier } \\
\mathrm{T} / \mathrm{Tx}\end{array}$ & N2 & MO & S1 \\
\hline \multirow[t]{2}{*}{$\begin{array}{l}\text { Estadio } \\
\text { IIC }\end{array}$} & $\begin{array}{c}\text { Cualquier } \\
T / T x\end{array}$ & N3 & MO & So \\
\hline & $\begin{array}{c}\text { Cualquier } \\
\text { T/Tx }\end{array}$ & N3 & Mo & S1 \\
\hline $\begin{array}{l}\text { Estadio } \\
\text { III }\end{array}$ & $\begin{array}{c}\text { Cualquier } \\
\text { T/Tx }\end{array}$ & $\begin{array}{c}\text { Cual- } \\
\text { quier N }\end{array}$ & M1a & Sx \\
\hline \multirow[t]{2}{*}{$\begin{array}{l}\text { Estadio } \\
\text { IIIA }\end{array}$} & $\begin{array}{l}\text { Cualquier } \\
\text { T/Tx }\end{array}$ & $\begin{array}{l}\text { Cual- } \\
\text { quier N }\end{array}$ & M1a & So \\
\hline & $\begin{array}{c}\text { Cualquier } \\
\mathrm{T} / \mathrm{Tx}\end{array}$ & $\begin{array}{l}\text { Cual- } \\
\text { quier N }\end{array}$ & M1a & S1 \\
\hline \multirow[t]{2}{*}{$\begin{array}{l}\text { Estadio } \\
\text { IIIB }\end{array}$} & $\begin{array}{l}\text { Cualquier } \\
\text { T/Tx }\end{array}$ & N1-N3 & MO & S2 \\
\hline & $\begin{array}{c}\text { Cualquier } \\
\text { T/Tx }\end{array}$ & $\begin{array}{l}\text { Cual- } \\
\text { quier N }\end{array}$ & M1a & S2 \\
\hline \multirow[t]{2}{*}{$\begin{array}{l}\text { Estadio } \\
\text { IIIC }\end{array}$} & $\begin{array}{c}\text { Cualquier } \\
T / T x\end{array}$ & N1-N3 & Mo & S3 \\
\hline & $\begin{array}{l}\text { Cualquier } \\
\text { T/Tx }\end{array}$ & $\begin{array}{l}\text { Cual- } \\
\text { quier N }\end{array}$ & M1a & S3 \\
\hline
\end{tabular}

minales $(90-95 \%)$ y del estroma gonadal $(5 \%) .^{15}$ De los primeros, de 30-60\% corresponden a seminomas, y el resto tienen más de un tipo celular puro. ${ }^{15}$
En general, el tratamiento médico-quirúrgico requiere la participación del urólogo, del patólogo y del oncólogo médico. El tratamiento con quimioterapia dependerá, estrictamente, de la estirpe histológica, del pronóstico y del seguimiento. ${ }^{16,17,18}$ En relación con el pronóstico, el Grupo Colaborador Internacional para el Cáncer de Células Germinales (International Germ Cell Cancer Collaborative Group, IGCCCG) definió un sistema de estadificación que incluye a los tumores seminomatosos y TCG-NS (no seminomatosos). ${ }^{19}$ Como consecuencia de los avances en el tratamiento de esta enfermedad, en las últimas décadas la supervivencia a cinco años de estos pacientes se ha incrementado, de $83 \%$ entre 1975-1977 a 97\% para 2005-2011.,3

El objetivo de este estudio fue: reportar las características epidemiológicas, histopatológicas y etiológicas del cáncer testicular en un hospital de tercer nivel en el periodo 2012-2017.

\section{MATERIALES Y MÉTODOS}

Estudio observacional, descriptivo y retrospectivo efectuado en pacientes con diagnóstico de cáncer testicular de cualquier estirpe histológica atendidos entre 2012 y 2017 en el servicio de Urología del Hospital General de México Dr. Eduardo Liceaga.

Criterios de inclusión: diagnóstico histopatológico postorquiectomía de cáncer testicular de cualquier estirpe histológica, seguimiento temporal a cinco años por clínica, marcadores tumorales y radiológico. Criterios de exclusión: no contar con diagnóstico de cáncer testicular. Criterios de eliminación: pérdida de seguimiento clínico, bioquímico y radiológico a cinco años.

La información de las variables cualitativas se resumió en frecuencias simples y relativas en porcentaje. La variabilidad de las frecuencias se obtuvo con IC95\%. En virtud de la distribución 
no normal de la edad se resumió en mediana y valores máximos y mínimos. La información se proporciona según la morfología del tumor. Para contrastar las diferencias entre los tipos de tumores según las variables analizadas se utilizó prueba de $\chi^{2}$ (variables cualitativas) y prueba de Kruskal-Wallis (variables cuantitativas). Los datos se analizaron con el paquete estadístico SPSS versión 20.0 y se consideró significación estadística a un valor de $\mathrm{p}<0.05$. Debido a que hubo grupos con menos de 10 participantes no se realizaron pruebas post-hoc.

\section{RESULTADOS}

Se analizaron los expedientes de 142 pacientes. Los tumores de células germinales mixtas y los seminomas clásicos fueron la estirpe histológica más frecuente: 44.4\% (63/142; IC95\%: 36.2 a $52.5 \%$ ) y $43.6 \%$ (62/142; IC95\%: 35.5 a $51.8 \%)$, respectivamente. El $12 \%$ restante se distribuyó en: teratomas maduros $1.4 \%$, teratomas inmaduros $4.2 \%$, linfomas de células $B$ grandes y difusas-LCBD 1.4\%, coriocarcinomas $1.4 \%$ y plasmocitomas $0.7 \%$. En total se registraron 77 pacientes $(54.2 \%)$ con tumores germinales no seminomatosos. Figura 1

El 50\% de los casos se registró en varones de 20 a 30 años con una media de 35.5. (Figura 2) En el análisis por tipo de cáncer sólo los linfomas aparecieron a una edad mayor (mediana 75 años).

Contrario a lo reportado en la bibliografía en relación con la lateralidad, en nuestro grupo hubo predominio izquierdo $(\mathrm{n}=80)(56.3 \%)$ vs derecho $(n=62)$, sin diferencia estadísticamente significativa para cada estirpe histológica.

\section{Cuadro 2}

En general, el estadio histopatológico más frecuente fue el T1 con $63.4 \%(n=90)$ seguido del T2 con $22.5 \%(n=32)$, T3 con $8.5 \%(n=12)$ y Tis con $4.9 \%(n=7)$; la diseminación local T4

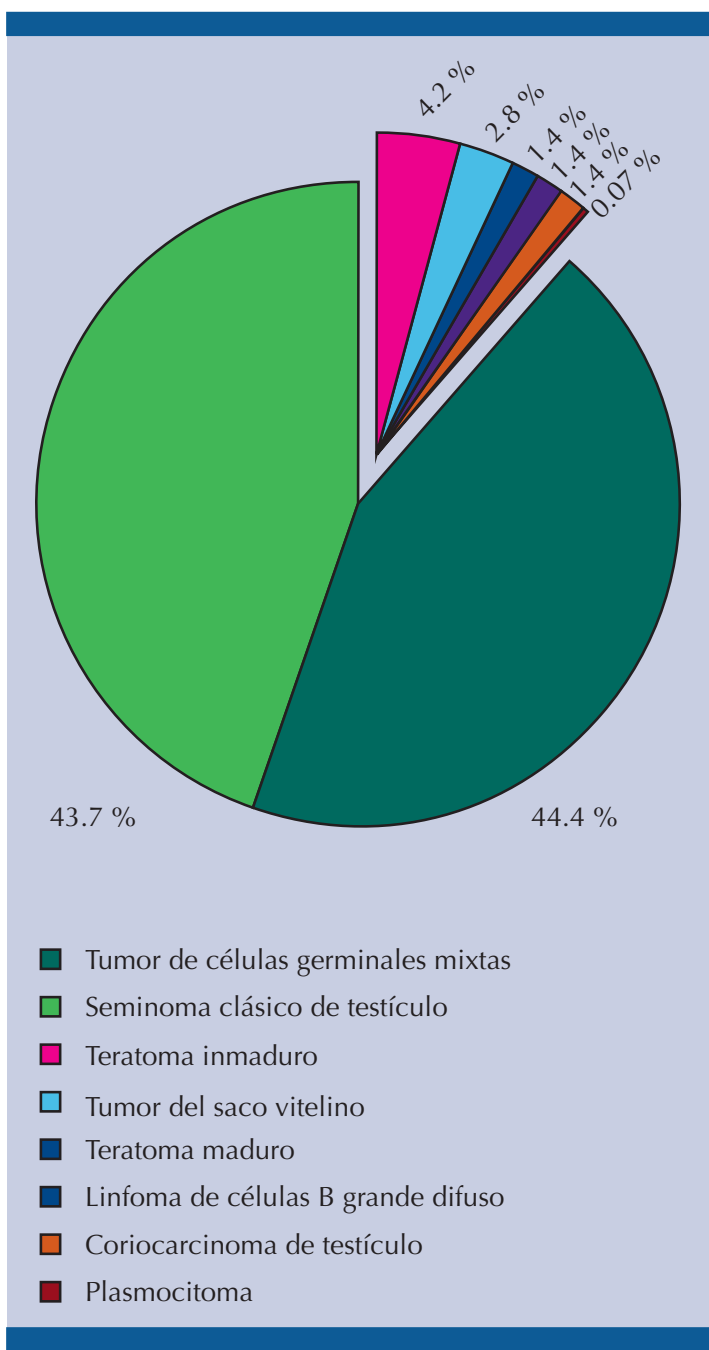

Figura 1. Distribución de los tumores testiculares según la estirpe histológica $(n=142)$.

sólo se observó en un caso (0.7\%). Este predominio de casos diagnosticados en estadio T1 y T2 no varió entre las estirpes tumorales.

Por lo que se refiere a la diseminación linfática (N) los tumores seminomatosos tuvieron una estadificación N0 en $80.6 \%$ de los casos ( $\mathrm{n}=$ 50) y $95.2 \%(n=59)$ no tuvieron mestástasis pulmonares u otras al momento del diagnóstico. La estirpe no seminomatosa (específicamente 
Cuadro 2. Comparación entre los diferentes tipos morfológicos de cáncer testicular

\begin{tabular}{|c|c|c|c|c|c|c|c|c|}
\hline \multirow[b]{2}{*}{ Variables } & \multirow{2}{*}{$\begin{array}{l}\text { Germinales } \\
(\mathrm{n}=62)\end{array}$} & \multicolumn{5}{|c|}{$\begin{array}{l}\text { Germinales no seminomatosos } \\
\qquad(\mathrm{n}=77)\end{array}$} & \multicolumn{2}{|c|}{$\begin{array}{c}\text { Otros } \\
(n=3) \\
\end{array}$} \\
\hline & & $\begin{array}{c}\text { Células } \\
\text { germinales } \\
\text { mixtas }(n=63)\end{array}$ & \begin{tabular}{|c|}
$\begin{array}{c}\text { Teratoma } \\
\text { inmaduro } \\
(n=6)\end{array}$ \\
\end{tabular} & $\begin{array}{l}\text { Tumor del } \\
\text { saco vitelino } \\
(n=4)\end{array}$ & $\begin{array}{c}\text { Teratoma } \\
\text { maduro } \\
(\mathrm{n}=2)\end{array}$ & $\begin{array}{l}\text { Coriocarcinoma } \\
\quad(n=2)\end{array}$ & $\begin{array}{l}\text { LCBD } \\
(n=2)\end{array}$ & $\begin{array}{l}\text { Plasmo- } \\
\text { citoma } \\
(n=1)\end{array}$ \\
\hline $\begin{array}{l}\text { Edad } \\
\text { (años) }\end{array}$ & 30.5 & 26 & 30.3 & 25.5 & 29.5 & 23 & 71.5 & 53 \\
\hline $\begin{array}{l}\text { Mediana } \\
\text { (min-máx) }\end{array}$ & $(19-54)$ & $(18-62)$ & $(21-50)$ & $(18-32)$ & $(29-30)$ & $(22-34)$ & $(70-73)$ & \\
\hline $\begin{array}{l}\text { Lateralidad } \\
\text { Izquierdo } \\
\text { Derecho }\end{array}$ & $\begin{array}{l}38(61.3 \%) \\
24(38.7 \%)\end{array}$ & $\begin{array}{l}35(55.6 \%) \\
28(44.4 \%)\end{array}$ & $\begin{array}{l}2(33.3 \%) \\
4(66.6 \%)\end{array}$ & $\begin{array}{l}2(50 \%) \\
2(50 \%)\end{array}$ & $\begin{array}{l}1(50 \%) \\
1(50 \%)\end{array}$ & $2(100 \%)$ & $\begin{array}{l}1(50 \%) \\
1(50 \%)\end{array}$ & $\begin{array}{c}1(100 \%) \\
-\end{array}$ \\
\hline Estadio & & & & & & & & \\
\hline Tis & $4(6.5 \%)$ & $2(3.2 \%)$ & - & - & - & $1(100 \%)$ & - & - \\
\hline $\mathrm{T} 1$ & $35(56.5 \%)$ & $44(69.8 \%)$ & $4(66.6 \%)$ & $2(50 \%)$ & $2(100 \%)$ & $1(100 \%)$ & 2 & - \\
\hline $\mathrm{T} 2$ & $18(29 \%)$ & $11(17.5 \%)$ & - & $2(50 \%)$ & - & - & $(100 \%)$ & $1(100 \%)$ \\
\hline T3 & $5(8.1 \%)$ & $6(9.5 \%)$ & $1(16.6 \%)$ & - & - & - & - & - \\
\hline T4 & - & - & $1(16.6 \%)$ & - & - & - & - & - \\
\hline No & $50(80.6 \%)$ & $36(57.2 \%)$ & 5 & $4(100 \%)$ & $2(100 \%)$ & $1(100 \%)$ & - & $1(100 \%)$ \\
\hline N1 & $12(19.4 \%)$ & $27(42.8 \%)$ & $(83.36 \%)$ & - & - & $1(100 \%)$ & - & - \\
\hline Mo & $59(95.2 \%)$ & $55(87.3 \%)$ & $1(16.6 \%)$ & $4(100 \%)$ & $1(50 \%)$ & - & 2 & $1(100 \%)$ \\
\hline M1A & $2(3.2 \%)$ & $7(11.1 \%)$ & 5 & - & - & $1(100 \%)$ & $(100 \%)$ & - \\
\hline M1B & $1(1.6 \%)$ & $1(1.6 \%)$ & $(83.36 \%)$ & - & $1(50 \%)$ & $1(100 \%)$ & $1(50 \%)$ & - \\
\hline So & $57(91.9 \%)$ & $44(69.8 \%)$ & $1(16.6 \%)$ & $2(50 \%)$ & - & - & $1(50 \%)$ & $1(100 \%)$ \\
\hline S1 & $3(4.8 \%)$ & $14(22.2 \%)$ & - & $1(25 \%)$ & $1(50 \%)$ & - & - & - \\
\hline S2 & $2(3.2 \%)$ & $4(6.4 \%)$ & $1(16.6 \%)$ & $1(25 \%)$ & $1(50 \%)$ & $1(100 \%)$ & $1(50 \%)$ & - \\
\hline S3 & - & $1(1.6 \%)$ & $\begin{array}{l}2(33.3 \%) \\
2(33.3 \%) \\
1(16.6 \%)\end{array}$ & - & - & $1(100 \%)$ & $1(50 \%)$ & - \\
\hline
\end{tabular}

Lateralidad: $\chi^{2}=5.41$ ( $7 \mathrm{gl}$ ) $\mathrm{p}=0.61$; edad: prueba de Kruskal-Wallis $\mathrm{p}=0.002$.

LCBD: linfoma de células B gigante difuso.

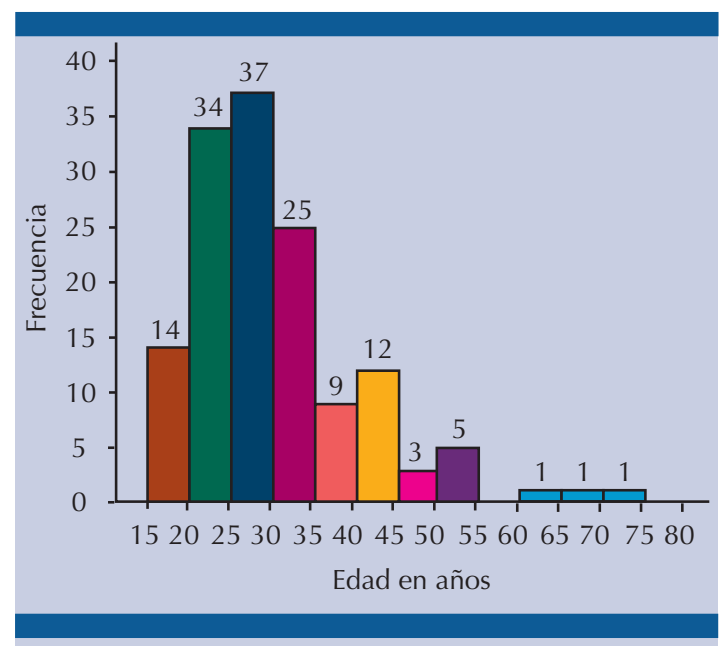

Figura 2. Distribución por edad de los pacientes con cáncer testicular $(\mathrm{n}=142)$. los tumores germinales de células mixtas) apareció en un estadio N0, M0 en $57.2 \%(n=36)$ y $87.3 \%(n=55)$ de los casos, respectivamente. El pronóstico basado en la clasificación de la IGCCCG se describe en el Cuadro 3; hubo casos con pronóstico bueno e intermedio en 91.9 y $1.6 \%$, respectivamente, en pacientes con tumores seminomatosos; $y$ un pronóstico bueno, intermedio y malo $(61 \%, 23.4,15.6 \%)$ para los tumores no seminomatosos. Cuadro 3

Al analizar los casos atendidos por año y, en particular para los tumores germinales de células mixtas y los seminomas, se observó mayor incidencia en los últimos dos años. Figura 3 
Cuadro 3. Distribución de pacientes según la clasificación pronóstica del cáncer testicular (IGCCCG).

\begin{tabular}{|l|c|c|}
\hline Pronóstico & Seminoma & $\begin{array}{c}\text { No } \\
\text { seminomatoso }\end{array}$ \\
\hline Buen pronóstico & $57(91.9 \%)$ & $47(61 \%)$ \\
\hline Pronóstico intermedio & $1(1.6 \%)$ & $18(23.4 \%)$ \\
\hline Mal pronóstico & & $12(15.6 \%)$ \\
\hline
\end{tabular}

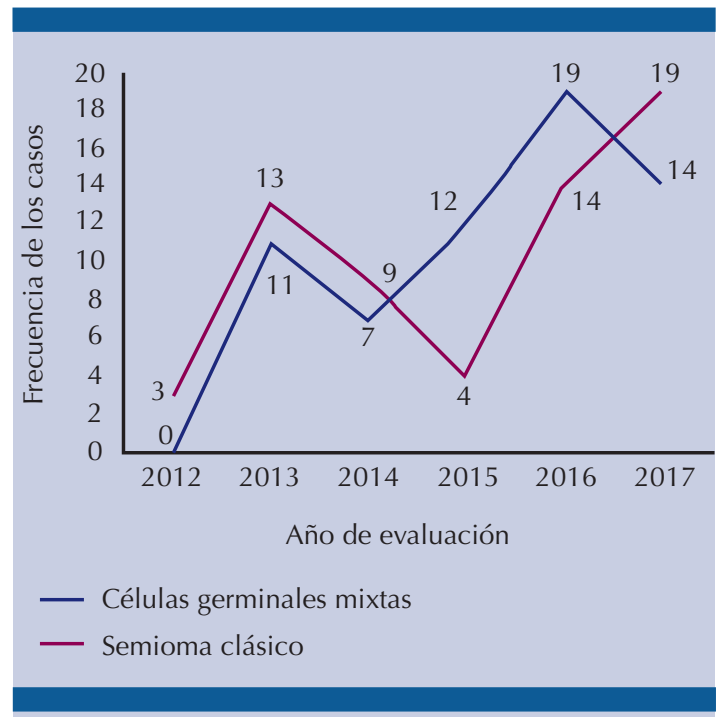

Figura 3. Incidencia de casos atendidos de 2012 a 2017 de tumores de células germinales mixtas ( $n=$ 63) y seminomas $(n=62)$.

\section{DISCUSIÓN}

Los tumores testiculares son las neoplasias más frecuentes del aparato genitourinario, de ahí la importancia de aportar a la experiencia la prevalencia del Hospital General de México en el último lustro.

Los porcentajes reportados difieren, en cuanto a la estirpe histológica y lateralidad de presentación del tumor testicular, de los informados por otros centros hospitalarios de concentración.7,10 Los porcentajes aquí informados son similares a los de Abomelha en el estudio Adult testicular cancer: Two decades of Saudi national data. ${ }^{20}$
De todos los cánceres testiculares incluidos en 20 años $(n=1004)$, los seminomas se registraron en $40.7 \%(n=408)$ y los no seminomas en $44.6 \%(n=596)$. Los subtipos de no seminomas registrados fueron: tumor mixto en $51.6 \%$ ( $n=$ 230), carcinoma embrionario en $19.9 \%$, tumor del saco vitelino en $12.3 \%$, germinomas en $6.7 \%$, teratomas en $6.0 \%$ y coriocarcinomas en $3.6 \% .{ }^{20}$ En otro estudio de Leveridge y colaboradores (2000- 2010), que incluyó 2650 pacientes, referente a la lateralidad, el testículo mayormente afectado fue el izquierdo en $51 \%$ de los casos $(n=1364),{ }^{10}$ porcentaje semejante a nuestros resultados.

De la información previa se desprende que la estirpe seminomatosa es la más prevalente; no obstante, en nuestro hospital existe una ligera prevalencia del tumor de células germinales mixtas y mayor aparición del tumor testicular en el lado izquierdo. Este dato coincidió con lo reportado por Leveridge y su grupo, ${ }^{10}$ puesto que no existe información previa en relación con la lateralidad y quizá esté asociado con casos de criptorquidia o mal descenso testicular subdiagnosticados. Con independencia de las características histopatológicas y la lateralidad de presentación del tumor testicular, los porcentajes determinados en esta investigación reflejan similitudes con respecto a las condiciones demográficas (edad de presentación) y grupos pronóstico reportados en la bibliografía mundial. ${ }^{2,3,4,7}$

\section{CONCLUSIONES}

En la población analizada el cáncer testicular es más frecuente entre los 20 y 30 años y, desde el punto de vista histológico, el cáncer germinal mixto registró mayor incidencia en los tumores puros seminomatosos, contrastante con lo reportado en la bibliografía y que puede estar directamente relacionado con el grupo etario atendido en el Hospital General de México. 
La manifestación clínica de los casos mostró preferencia por la lateralidad izquierda, quizá asociada con casos no diagnosticados de criptorquidia o atrofia testicular. Se trató de una variable no contemplada en el diseño inicial de este estudio y que deberá incluirse en ensayos futuros con modelos de investigación distintos para asociar causalidad.

En general, el estudio aquí comunicado es consistente con lo reportado en la bibliografía pero las discretas diferencias abren un panorama para replantear y analizar los motivos de causalidad de estas formas clínicas e histológicas de presentación. Hace falta reunir más casos y recurrir a otro tipo de análisis de datos para dilucidar las características de los pacientes que acuden al Hospital General de México y determinar los factores asociados con la recurrencia y pronóstico de este padecimiento oncológico con auge emergente.

\section{REFERENCIAS}

1. Coursey-Moreno C, et al. Testicular tumors: what radiologists need to know-differential diagnosis, staging, and management. Radiographics 2015;35(2):400-15. https:// doi.org/10.1148/rg.352140097

2. Jiménez-Rios M. Panorama epidemiológico del cáncer genitourinario en la Zona Centro de México. Rev Mex Urol 2011;71(S3): 3-6.

3. Albers P, et al. Guidelines on Testicular Cancer: 2015 Update. European Urology 2015;68(6): 1054-68. https://doi. org/10.1016/j.eururo.2015.07.044

4. Walsh TJ. Incidence of testicular germ cell cancers in U.S. children: SEER program experience 1973 to 2000. Urology 2006; 68(2):402-5. https://doi.org/10.1016/j. urology.2006.02.045

5. Gómez A, et al. Incidencia de cáncer en una Unidad de atención Oncológica del Instituto Mexicano del Seguro Social (IMSS) en Toluca, Estado de México. Rev Gac Med Mex 2014; 150(2):297-303.

6. Nigam M, et al. Increasing incidence of testicular cancer in the United States and Europe between 1992 and 2009. World Journal of Urology 2015;33(5):623-31. https://doi. org/10.1007/s00345-014-1361-y

7. Lindsey AT, et al. Cancer statistics for Asian Americans, Native Hawaiians, and Pacific Islanders, 2016: Converging incidence in males and females. Cancer J Clin 2016;66(1):730. https://doi.org/10.3322/caac.21335

8. Zachary L. Testicular Cancer Epidemiology, Diagnosis, and Management. Med Clin N Am 2018; 251-64. doi: 10.1016/j. mcna.2017.10.003.

9. Hemminki $\mathrm{K}$, et al. Familial risk in testicular cancer as a clue to a heritable and environmental aetiology. $\mathrm{Br}$ J Cancer 2004; 90: 1765-70. https://doi.org/10.1038/ sj.bjc. 6601714

10. Leveridge MJ, et al. Temporal trends in management and outcomes of testicular cancer: A Population-Based Study. Cancer J 2018;124(13):2724-32. doi: 10.1002/cncr.31390.

11. Shanmugalingam $T$, et al. Global incidence and outcome of testicular cancer. Clin Epidemiol 2013; 5: 417-27. doi: $10.2147 /$ CLEP.S34430

12. Dogra VS, et al. Sonography of the scrotum. Radiology 2003;227(1): 18-36. doi 10.1148/radiol.2271001744

13. See $\mathbf{W}$, et al. Chest staging in testis cancer patients: imaging modality selection based upon risk assessment as determined by abdominal computerized tomography scan results. J Urol 1993;150(3):874-78. https://doi.org/10.1016/ S0022-5347(17)35637-9

14. Chia VM, et al. International trends in the incidence of testicular cancer, 1973-2002. Cancer Epidemiol Biomarkers Prev 2010; 19:1151-59. doi: 10.1158/1055-9965. EPI-10-0031

15. Rajpert-De Meyts E. et al. Testicular germ cell tumours. Lancet 2016;387(10029):1762-74. doi: 10.1016/S01406736(15)00991-5.

16. Beck SDW, et al. Pathologic findings and therapeutic outcome of desperation post-chemotherapy retroperitoneal lymph node dissection in advanced germ cell cancer. Urol Oncol 2005;23(6):423-30. doi:10.1016/j. urolonc.2005.06.007

17. Beck SD, et al. Teratoma in the orchiectomy specimen and volume of metastasis are predictors of retroperitoneal teratoma in postchemotherapy nonseminomatous testis cancer. J Urol 2002;168(4 Pt 1):1402-4. doi: 10.1097/01. ju.0000027902.28824.71

18. Travis LB, et al. Second cancers among 40,576 testicular cancer patients: focus on long term survivors. J Natl Cancer Inst 2005;97(18):1354-65. https://doi.org/10.1093/ jnci/dji278

19. International Germ Cell Consensus Classification: a prognostic factor-based staging system for metastatic germ cell cancers. International Germ Cell Cancer Collaborative Group. J Clin Oncol 1997;15(2):594-603. http://hdl.handle. net/10541/94716

20. Mohamed A. Adult testicular cancer: Two decades of Saudi national data. Urol Ann 2017;9(4):305-9. doi: 10.4103/ UA.UA 1117 